\title{
Unperturbed Chain Dimension of Branched Polypeptides. I. Star-Like Polypeptides
}

\author{
Masahito OKa and Akio NaKaJima \\ Department of Polymer Chemistry, Kyoto University, Sakyo-ku, \\ Kyoto 606, Japan.
}

(Received May 7, 1977)

\begin{abstract}
Theoretical calculations for the mean square of unperturbed radius of gyration $\left\langle S^{2}\right\rangle_{0, a v}$ and the branching factor $g$ of star-like polypeptides in random coil form were carried out for two cases in which the degree of polymerization of the subchains distributed uniformly and randomly. The radius of the gyration ratio $\left\langle S^{2}\right\rangle_{0, a v} / n l^{2}$ and $g$ depended on the degree of polymerization as well as the number of branches, and increased with an increasing degree of polymerization.
\end{abstract}

KEY WORDS Star-Like Chain / Branched Polypeptides / Unperturbed Dimension / Radius of Gyration / Branching Factor / Uniform Distribution / Random Distribution /

Some natural proteins have cross-linked structure through the $\mathbf{S}-\mathbf{S}$ bond between cystine residues. Proteins, even when they have no cross-linked structure, have complicated highorder structures owing to intramolecular interactions. Therefore, we may consider that such proteins have similar structures to the crosslinked polymers. Consequently, using branched polypeptide as a model of natural protein is reasonable. By this model, we may determine step by step the complicated intramolecular interactions in proteins.

As a kind of branched polypeptides, there are star-like polypeptides, having branches stemming from one point. These star-like polypeptides are similar to globular proteins in structures. Thus these polypeptides may provide new information about globular proteins.

Unperturbed dimension is one of the fundamental molecular properties for investigating the form of the polymer chains in dilute solution. Unperturbed dimensions of the branched polymer chains have been calculated using the Gaussian chain approximation by Zimm and Stockmayer, ${ }^{1}$ and Kurata and Fukatsu. ${ }^{2}$ The degree of polymerization for many globular proteins is in a range of 50-200. The method of the Gaussian chain approximation is therefore not always adequate, as this method is useful for infinite degree of polymerization. Flory and his coworkers $^{3-9}$ have calculated the dimension of polymer chains as a function of the degree of polymerization for linear polymer chains. These calculations were carried out on the basis of the rotational isomeric model. In this paper, Flory's method is applied to the star-like polypeptide chains for calculating the unperturbed dimensions.

\section{MEAN SQUARE OF THE RADIUS OF GYRATION}

In Figure 1, a star-like chain molecule is shown.

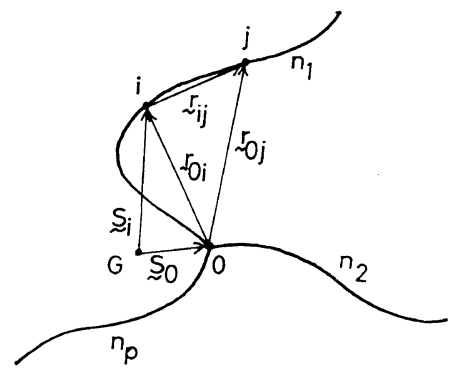

Figure 1. A star-like chain molecule. 
This chain possesses $p$ branches (subchains) attached to one branching point 0 . The degree of polymerization of the $k$-th subchain is assumed to be $n_{k}$. The branching point is given the symbol 0 , and each atom (structural unit) in the $k$-th subchain is counted as $1,2, \ldots$, and $n_{k}$ toward the end of the subchain. The vector from the center of mass $G$ of the molecule to the $i$-th atom of the subchain is $S_{i}$. As each atom is assumed to be equal in mass, the square radius of gyration $S^{2}$ is represented by

$$
S^{2}=\left(\sum_{k=1}^{p} \sum_{i=1}^{n_{k}} S_{i}{ }^{2}+S_{0}{ }^{2}\right)(n+1)^{-1}
$$

If the vector from the $i$-th atom to the $j$-th atom is $r_{i j}$, then

$$
\begin{aligned}
S^{2}= & {\left[\sum_{1 \leqq k<h \leqq p}^{2} \sum_{0 \leqq i<j \leqq n_{l k h}}^{2} r_{i j}^{2}\right.} \\
& \left.-(p-2) \sum_{t=1}^{p} \sum_{0 \leqq i<j \leqq n_{t}}^{2} r_{i j}^{2}\right](n+1)^{-2}
\end{aligned}
$$

from the theorem of Lagrange (See Appendix), where $n_{n k}$ is represented by $n_{k h}=n_{k}+n_{h}$ and symbolism of the type $\Sigma^{k}$ indicates the serial $k$ symbols of summation. The amide bond in polypeptide chains has a planar trans-partial double bond, so that the chain is regarded as a sequences of virtual bonds between the adjacent $\alpha$-carbon atoms. Such a character of polypeptide chain makes it possible to assume that the rotation of the virtual bond is treated as if it does not depend on the rotation of its adjacent bonds. Furthermore, it may be assumed that the rotation of the virtual bonds succeding the branching point is treated as an independent rotation. Thus one obtains

$$
\begin{aligned}
\left\langle S^{2}\right\rangle_{0}= & {\left[\sum_{1 \leqq k<h \leqq p}^{2} \sum_{0 \leqq i<j \leqq n_{k h}}^{2}\left\langle r_{i j}^{2}\right\rangle\right.} \\
& \left.-(p-2) \sum_{t=1}^{p} \sum_{0 \leqq i<j \leqq n_{t}}^{2}\left\langle r_{i j}^{2}\right\rangle\right](n+1)^{-2}
\end{aligned}
$$

where the suffix 0 indicates the unperturbed state. Furthermore if all subchains are statistically equal, eq 3 can be simplified as

$$
\begin{aligned}
\left\langle S^{2}\right\rangle_{0}= & {\left[\frac{p(p-1)}{2} \sum_{0 \leqq i<j \leqq n_{k h}}\left\langle r_{i j}^{2}\right\rangle\right.} \\
& \left.-p(p-2) \sum_{0 \leqq i<j \leqq n_{k}}^{2}\left\langle r_{i j}^{2}\right\rangle\right](n+1)^{-2}
\end{aligned}
$$

For a linear chain whose rotation of the bond is treated as an independent rotation, the value

$$
\left\langle S^{2}\right\rangle_{0}=(n+1)^{-2} \sum_{0 \leqq i<j \leqq n}^{2}\left\langle r_{i j}^{2}\right\rangle
$$

is represented by two methods.

One is the method (method I) to represent $\left\langle S^{2}\right\rangle_{0}$ as a series of matrix $\langle\boldsymbol{T}\rangle$ by applying the regular property of matrix $\langle\boldsymbol{T}\rangle$, where $\langle\boldsymbol{T}\rangle$ is the statistical average of the matrix $T$ which transform the representation of a vector in the coordinate system of bond $i+1$ into its representation in a similar coordinate system affixed to bond $i$ (see ref 3). In this method, $\left\langle S^{2}\right\rangle_{0}$ is represented by eq 6 ,

$$
\begin{aligned}
\left\langle S^{2}\right\rangle_{0}= & l^{2}(n+1)^{-2} \\
& \times\left\{\frac{1}{6} n(n+1)(n+2)\left[\left(\boldsymbol{E}_{3}+\langle\boldsymbol{T}\rangle\right)\left(\boldsymbol{E}_{3}-\langle\boldsymbol{T}\rangle\right)^{-1}\right]_{11}\right. \\
& -n(n+1)\left[\left(\boldsymbol{E}_{3}-\langle\boldsymbol{T}\rangle\right)^{-2}\langle\boldsymbol{T}\rangle\right]_{11} \\
& +2 n\left[\left(\boldsymbol{E}_{3}-\langle\boldsymbol{T}\rangle\right)^{-3}\langle\boldsymbol{T}\rangle^{2}\right]_{11} \\
& \left.-2\left[\left(\boldsymbol{E}_{3}-\langle\boldsymbol{T}\rangle\right)^{-4}\langle\boldsymbol{T}\rangle^{3}\left(\boldsymbol{E}_{3}-\langle\boldsymbol{T}\rangle^{n}\right)\right]_{11}\right\} \quad \text { (6) }
\end{aligned}
$$

where the subscript 11 of $[\boldsymbol{M}]_{11}$ denotes the 1, 1-element of matrix $\boldsymbol{M}$, the matrix $\boldsymbol{E}_{k}$ represents the unit matrix of the order $k, l$ is the length between successive $\alpha$-carbons, and $n$ is the number of virtual bonds.

The other is Flory-Jernigan ${ }^{7}$ matrix method (method II). In this method, $\left\langle S^{2}\right\rangle_{0}$ is represented by eq 7 .

$$
\left\langle S^{2}\right\rangle_{0}=2(n+1)^{-2} \boldsymbol{J}_{7}^{*} \boldsymbol{D}_{1}^{(n)} \boldsymbol{J}_{7}
$$

where

$$
\boldsymbol{D}_{i}=\left[\begin{array}{ccccc}
1 & 1 & \boldsymbol{l}^{t}\langle\boldsymbol{T}\rangle & l^{2} / 2 & l^{2} / 2 \\
0 & 1 & \boldsymbol{l}^{t}\langle\boldsymbol{T}\rangle & l^{2} / 2 & l^{2} / 2 \\
\mathbf{0} & \mathbf{0} & \langle\boldsymbol{T}\rangle & \boldsymbol{l} & \boldsymbol{l} \\
0 & 0 & \mathbf{0} & 1 & 1 \\
0 & 0 & \mathbf{0} & 0 & 1
\end{array}\right]_{i}
$$

$J_{k}{ }^{*}$ is defined as the row vector consisting of $k$ elements, the first element being unity and all succeeding elements being zero, and $\boldsymbol{J}_{k}$ is defined as the column vector consisting of $k$ elements, the last element being unity and other element being zero. Symbolism of the type $D_{1}{ }^{(n)}$ (see ref 3 ) indicates the serial product of the type $D_{1} D_{2} \cdots D_{n}$, i.e., a product of $n$ such terms. $\quad 0$ is rectangular null matrix of the appropriate dimension, and $l$ is the bond vector as a column. 
Unperturbed Chain Dimension of Branched Polypeptides. I.

\section{RADIUS OF THE GYRATION RATIO OF \\ STAR-LIKE POLYPEPTIDES WITH \\ UNIFORM AND RANDOM DISTRIBUTION IN \\ LENGTH}

Considering eq 5 and 6 , or eq 5 and 7 , the unperturbed dimension of star-like chains whose degree of polymerization are known can be calculated by using eq 3 or 4 . However it seems almost impossible to determine the degree of polymerization for all subchains. So the average is carried out in two statistically interesting cases. One case is the average of the uniform distribution of subchain length. The other is the case in which the average of the random distribution of subchain length.

Uniform Distribution of Subchain Length

Let the degree of polymerization of the molecule be $n$, and that of the $k$-th subchain be $n_{k}$; then, $n_{l}$ is represented by

$$
n_{k}=n / p \quad(k=1,2, \cdots, p)
$$

In the case of uniform distribution of subchain lengths, using eq 9 we obtain eq 10 from 4 .

$$
\begin{aligned}
\left\langle S^{2}\right\rangle_{0, \mathrm{av}}= & (n+1)^{-2}\left[\frac{p(p-1)}{2} \sum_{0 \leqq i<j \leqq 2 n_{l}}^{2}\left\langle r_{i j}^{2}\right\rangle\right. \\
& \left.-p(p-2) \sum_{0 \leqq i<j \leqq n_{k}}\left\langle r_{i j}^{2}\right\rangle\right]
\end{aligned}
$$

The subscript av of $\left\langle S^{2}\right\rangle_{0 \text {,av }}$ denotes the average on the distribution of the degree of polymerization of subchains. Using method I (eq 5 and $6)$, we obtain eq 11 .

$$
\begin{aligned}
\left\langle S^{2}\right\rangle_{0, \mathrm{a} \mathrm{v}}= & n l^{2}\left\{\frac{1}{6} \frac{(n+p)[(3 p-2) n+2 p]}{p^{2}(n+1)^{2}}\right. \\
& \times\left[\left(\boldsymbol{E}_{3}-\langle\boldsymbol{T}\rangle\right)^{-1}\left(\boldsymbol{E}_{3}+\langle\boldsymbol{T}\rangle\right)\right]_{11} \\
& -\frac{1}{(n+1)}\left[\left(\boldsymbol{E}_{3}-\langle\boldsymbol{T}\rangle\right)^{-2}\langle\boldsymbol{T}\rangle\right]_{11} \\
& +\frac{2}{(n+1)^{2}}\left[\left(\boldsymbol{E}_{3}-\langle\boldsymbol{T}\rangle\right)^{-3}\langle\boldsymbol{T}\rangle^{2}\right]_{11} \\
& +\frac{p}{n(n+1)^{2}}\left[( \boldsymbol { E } _ { 3 } - \langle \boldsymbol { T } \rangle ) ^ { - 4 } \langle \boldsymbol { T } \rangle ^ { 3 } \left\{(p-3) \boldsymbol{E}_{3}\right.\right. \\
& \left.\left.\left.-2(p-2)\langle\boldsymbol{T}\rangle^{n / p}+(p-1)\langle\boldsymbol{T}\rangle^{2 n / p}\right\}\right]_{11}\right\}
\end{aligned}
$$

Using method II (eq 5 and 7), we obtain eq 12.

$$
\begin{aligned}
\left\langle S^{2}\right\rangle_{0, \mathrm{av}}= & (n+1)^{-2}\left[p(p-1) \boldsymbol{J}_{7}{ }^{*} \boldsymbol{D}_{1}{ }^{(2 n / p)} \boldsymbol{J}_{7}\right. \\
& \left.-2 p(p-2) \boldsymbol{J}_{7}{ }^{*}{\boldsymbol{D}_{1}}^{(n / p)} \boldsymbol{J}_{7}\right]
\end{aligned}
$$

Equations 11 and 12 are derived only by using the rotational isomeric approximation, and are applied to all star-like chains whose branches have the same degree of polymerization. Substituting 1 or 2 for $p$ in eq 11 and 12, we obtain the equations for linear chain by simple calculation.

\section{Random Distribution of Subchain Lengths}

When the degree of polymerization of the subchains are randomly distributed, the average of the mean square of radius of gyration is obtained by the following equation.

$$
\left\langle S^{2}\right\rangle_{0, \mathrm{av}}=\sum_{0<N_{1}<\cdots<N_{p-1}<n}\left\langle S^{2}\right\rangle_{0} / \sum_{0<N_{1}<\cdots<N_{p-1}<n} \sum^{p-1} 1
$$

where

$$
N_{k}=\sum_{t=1}^{k} n_{t}
$$

In the case of method I, according to from eq 4-6,

$$
\begin{aligned}
\left\langle S^{2}\right\rangle_{0}= & l^{2}(n+1)^{-2}\left\{\frac{1}{6}[n(n+1)(n+2)\right. \\
& \left.-6 \sum_{1 \leqq k<l<m \leqq p}^{3} n_{l k} n_{l} n_{m}\right] \\
& \times\left[\left(\boldsymbol{E}_{3}-\langle\boldsymbol{T}\rangle\right)^{-1}\left(\boldsymbol{E}_{3}+\langle\boldsymbol{T}\rangle\right)\right]_{11} \\
& -n(n+1)\left[\left(\boldsymbol{E}_{3}-\langle\boldsymbol{T}\rangle\right)^{-2}\langle\boldsymbol{T}\rangle\right]_{11} \\
& +2 n\left[\left(\boldsymbol{E}_{3}-\langle\boldsymbol{T}\rangle\right)^{-3}\langle\boldsymbol{T}\rangle^{2}\right]_{11} \\
& +\left[\left\{p(p-3) \boldsymbol{E}_{3}+2 \sum_{1 \leqq k<h \leqq p}^{2}\langle\boldsymbol{T}\rangle^{n_{k h}}\right.\right. \\
& \left.\left.\left.-2(p-2) \sum_{k=1}^{p}\langle\boldsymbol{T}\rangle^{n_{k}}\right\}\left(\boldsymbol{E}_{3}-\langle\boldsymbol{T}\rangle\right)^{-4}\langle\boldsymbol{T}\rangle^{3}\right]_{11}\right\}
\end{aligned}
$$

Using eq 13 and 15 , we obtain eq 16 .

$$
\begin{aligned}
\left\langle S^{2}\right\rangle_{0, \text { av }}= & n l^{2}\left\{\frac{1}{6} \frac{6 p}{(p+1)(p+2)} \frac{(n+2)}{(n+1)}\right. \\
& \times\left[\left(\boldsymbol{E}_{3}-\langle\boldsymbol{T}\rangle\right)^{-1}\left(\boldsymbol{E}_{3}+\langle\boldsymbol{T}\rangle\right)\right]_{11} \\
& -\frac{1}{n+1}\left[\left(\boldsymbol{E}_{3}-\langle\boldsymbol{T}\rangle\right)^{-2}\langle\boldsymbol{T}\rangle\right]_{11} \\
& +\frac{2}{(n+1)^{2}}\left[\left(\boldsymbol{E}_{3}-\langle\boldsymbol{T}\rangle\right)^{-3}\langle\boldsymbol{T}\rangle^{2}\right]_{11} \\
& +p !(p-1)(-1)^{p}\left[\left(\boldsymbol{E}_{3}-\langle\boldsymbol{T}\rangle\right)^{-p-2}\langle\boldsymbol{T}\rangle^{n+3}\right]_{11} \\
& \times\left[\prod_{k=-1}^{p-2}(n-k)(n+1)\right]^{-1} \\
& +(p+1) !(p-2)(-1)^{p} \\
& \times\left[\left(\boldsymbol{E}_{3}-\langle\boldsymbol{T}\rangle\right)^{-p-3}\langle\boldsymbol{T}\rangle^{n+3}\right]_{11}
\end{aligned}
$$


M. OKA and A. NAKAJIMA

$$
\begin{aligned}
& \times\left[\prod_{k=-1}^{p-1}(n-k)(n+1)\right]^{-1} \\
& +p ! \sum_{i=0}^{p-1}(p-1) i+p-3 \\
& \times\left[\left(E_{3}-\langle T\rangle\right)^{-i-4}\langle\boldsymbol{T}\rangle^{i+3}\right]_{11} \\
& \left.\times\left[\prod_{k=-1}^{i}(n-k)(n+1)\right]^{-1}\right\}
\end{aligned}
$$

To calculate the random average of eq 15, following relations are used,

$$
\begin{aligned}
& \sum_{0<N_{1}<\cdots<N_{p-1}<n}^{p-1} n_{k} n_{l} n_{m}=\frac{1}{(p+2) !} \prod_{i=-2}^{p-1}(n-i) \\
& \sum_{0<N_{1}<\cdots<N_{p-1}<n}^{p-1} \quad 1=\frac{1}{(p-1) !} \prod_{i=1}^{p-1}(n-i) \\
& \sum_{0<N_{1}<\cdots<N_{p-1}<n}^{p-1} \boldsymbol{A}^{n_{k}} \\
& =(-1)^{p-1}\left(\boldsymbol{E}_{m}-\boldsymbol{A}\right)^{1-p} \boldsymbol{A}^{n}+(-1)^{p}\left(\boldsymbol{E}_{m}-\boldsymbol{A}\right)^{1-p} \boldsymbol{A}^{p-1} \\
& +\sum_{j=2}^{p-1} \prod_{k=j}^{p-1} \frac{(n-k)}{(p-j) !}\left(\boldsymbol{E}_{m}-\boldsymbol{A}\right)^{1-j} \boldsymbol{A}^{j-1}(-1)^{j} \quad(18) \\
& \sum^{p-1} \boldsymbol{A}^{n_{k}+n_{h}} \\
& =(n-p+1)\left(\boldsymbol{E}_{m}-\boldsymbol{A}\right)^{2-p} \boldsymbol{A}^{n}(-1)^{p} \\
& \quad+(p-2)\left(\boldsymbol{E}_{m}-\boldsymbol{A}\right)^{1-p} \boldsymbol{A}^{n}(-1)^{p} \\
& \quad+\left(\boldsymbol{E}_{m}-\boldsymbol{A}\right)^{1-p} \prod_{k=1}^{p-1}(n-k) \\
& \quad \times \sum_{j=2}^{p-1}(p-3) ! \\
& \sum_{p-3} C_{p-j-1}
\end{aligned}
$$

where $\boldsymbol{A}$ represents the regular matrix of the order $m$.

In the case of method II, we define $\left\langle A_{p, k}\right\rangle$ and $\left\langle B_{p, k h}\right\rangle$ by eq 20 .

$$
\begin{aligned}
\left\langle A_{p, k}\right\rangle & =\boldsymbol{J}_{7}{ }^{*} \boldsymbol{D}_{1}{ }^{\left(n_{k}\right)} \boldsymbol{J}_{7} \\
\left\langle\boldsymbol{B}_{p, k h}\right\rangle & =\boldsymbol{J}_{7}^{*} \boldsymbol{D}_{1}^{\left(n_{k}+n_{h}\right)} \boldsymbol{J}_{7}
\end{aligned}
$$

Using eq 4, 5, 7, and 20, we obtain

$$
\left\langle S^{2}\right\rangle_{0}=(n+1)^{-2}\left[p(p-1)\left\langle B_{p, k h}\right\rangle-2 p(p-2)\left\langle A_{p, k}\right\rangle\right]
$$

Substitution eq 21 into 13, we obtain

$$
\begin{aligned}
\left\langle S^{2}\right\rangle_{0, \mathrm{av}}= & (n+1)^{-2}\left[p(p-1)\left\langle B_{p}\right\rangle_{\mathrm{av}}\right. \\
& \left.-2 p(p-2)\left\langle A_{p}\right\rangle_{\mathrm{av}}\right]
\end{aligned}
$$

where $\left\langle A_{p}\right\rangle_{\mathrm{av}}$ and $\left\langle B_{p}\right\rangle_{\mathrm{av}}$ are defined as random average of $\left\langle A_{p, k}\right\rangle$ and $\left\langle B_{p, k h}\right\rangle$, respectively.

Calculation of $\left\langle A_{p}\right\rangle_{\mathrm{av}}$ is carried out as follows. In the case of $p=3$, we let

$$
a_{2}=\sum_{0<N_{1}<N_{2}} \boldsymbol{J}_{7}^{*} \boldsymbol{D}_{1}^{\left(N_{1}\right)} \boldsymbol{J}_{7}
$$

The sum in this equation is obtained by using the following matrix $\{\boldsymbol{H}(2)\}_{i}$

$$
\{\boldsymbol{H}(2)\}_{i}=\left[\begin{array}{cc}
\boldsymbol{D} & \boldsymbol{D J}_{\mathbf{7}} \\
\mathbf{0} & 1
\end{array}\right]_{i}
$$

As shown in eq 27, $\{\boldsymbol{H}(k)\}_{i}$ represents a series of expanded matrices whose starting matrix is D. $\{\boldsymbol{H}(2)\}_{i}$ in eq 23 represents the 2 nd matrix of this series. Thus,

$$
\boldsymbol{a}_{2}=\boldsymbol{J}_{8}{ }^{*}\{\boldsymbol{H}(2)\}_{1}^{\left(N_{2}-1\right)} \boldsymbol{J}_{8}
$$

Similarly, we let

$$
a_{3}=\sum_{1<N_{2}<n} a_{2}
$$

and using the following matrix,

$$
\{\boldsymbol{H}(3)\}_{i}=\left[\begin{array}{cc}
\boldsymbol{H}(2) & \boldsymbol{H}(2) \boldsymbol{J}_{8} \\
\mathbf{0} & \mathbf{1}
\end{array}\right]_{i}
$$

we obtain the summation in eq 24 .

$$
\boldsymbol{a}_{3}=\boldsymbol{J}_{9}^{*}\{\boldsymbol{H}(3)\}_{1}^{(n-2)} \boldsymbol{J}_{9}
$$

From the definition of $\left\langle A_{3}\right\rangle_{a v}$, we obtain

$$
\left\langle A_{3}\right\rangle_{\mathrm{av}}=a_{3} / \sum_{0<N_{1}<N_{2}<n}^{2} 1
$$

Substitution of eq 25 into this equation gives

$$
\left\langle A_{3}\right\rangle_{\mathrm{av}}=\boldsymbol{J}_{9}^{*}\{\boldsymbol{H}(3)\}_{1}{ }^{(n-2)} \boldsymbol{J}_{9} / \sum_{0<N_{1}<N_{2}<n}^{2} 1
$$

Similarly, we obtain $\left\langle A_{p}\right\rangle_{\mathrm{av}}$

$$
\left\langle A_{p}\right\rangle_{\mathrm{av}}=\left.\boldsymbol{J}_{p+6}^{*}\{\boldsymbol{H}(p)\}_{1}^{(n-p+1)} \boldsymbol{J}_{p+6}\right|_{0<N_{1}<\cdots<N_{p-1}<n} \sum^{p-1} 1
$$

where

$$
\begin{aligned}
\{\boldsymbol{H}(k)\}_{i} & =\left[\begin{array}{cc}
\boldsymbol{H}(k-1) & \boldsymbol{H}(k-1) \boldsymbol{J}_{k+5} \\
\mathbf{0} & 1
\end{array}\right]_{i} \quad(k \geq 2) \\
\{\boldsymbol{H}(\mathbf{1})\}_{i} & =\boldsymbol{D}_{i}
\end{aligned}
$$

Calculation of $\left\langle B_{p}\right\rangle_{a v}$ is carried out in the following way. In the case of $p=4$, we let

$$
b_{3}=\sum_{0<N_{1}<N_{2}<N_{3}}^{2} \boldsymbol{J}_{7}^{*} \boldsymbol{D}_{1}^{\left(N_{1}\right)} \boldsymbol{D}_{N_{1}+1}^{\left(N_{2}-N_{1}\right)} J_{7}
$$

The sum in this equation is obtained by using the following matrix

$$
\begin{aligned}
\{\boldsymbol{K}(2)\}_{i} & =\left[\begin{array}{cc}
\boldsymbol{D} & \boldsymbol{I}_{0} \boldsymbol{H}(2) \\
\mathbf{0} & \boldsymbol{H}(2)
\end{array}\right]_{i} \\
\{\boldsymbol{K}(2)\}_{1} & =\left[\begin{array}{ll}
\boldsymbol{D}_{1} & \mathbf{0}
\end{array}\right]
\end{aligned}
$$

where 
Unperturbed Chain Dimension of Branched Polypeptides. I.

$$
I_{0}=\left[\begin{array}{ll}
E_{7} & 0
\end{array}\right]
$$

and orders of matrices $\{\boldsymbol{K}(2)\}_{1}$ and $\boldsymbol{I}_{0}$ are respectively, $7 \times 15$ and $7 \times 8$. Thus, we obtain

$$
b_{3}=\boldsymbol{J}_{7}^{*}\{\boldsymbol{K}(2)\}_{1}^{(n-1)} \boldsymbol{J}_{15}
$$

Similarly, we let

$$
b_{4}=\sum_{2<N_{3}<n} b_{3}
$$

and using the following matrices,

$$
\begin{aligned}
& \{\boldsymbol{K}(3)\}_{i}=\left[\begin{array}{cc}
\boldsymbol{K}(2) & \boldsymbol{K}(2) \boldsymbol{J}_{15} \\
\mathbf{0} & 1
\end{array}\right]_{i} \quad(i \geq 2) \\
& \{\boldsymbol{K}(3)\}_{1}=\left[\begin{array}{ll}
\boldsymbol{D}_{1} & \mathbf{0}
\end{array}\right]
\end{aligned}
$$

we obtain $b_{4}$ as

$$
b_{4}=\boldsymbol{J}_{7}^{*}\{\boldsymbol{K}(3)\}_{1}^{(n-2)} \boldsymbol{J}_{16}
$$

where the order of $\{\boldsymbol{K}(3)\}_{1}$ is $7 \times 16$. From the definition of $\left\langle B_{4}\right\rangle_{\mathrm{av}}$ and eq 29

$$
\left\langle B_{4}\right\rangle_{\mathrm{av}}=\boldsymbol{J}_{7}{ }^{*}\{\boldsymbol{K}(3)\}_{1}{ }^{(n-2)} \boldsymbol{J}_{16} / \sum_{0<N_{1}<N_{2}<N_{3}<n} 1
$$

Similarly, we obtain $\left\langle B_{p}\right\rangle_{\text {av }}$

$$
\begin{aligned}
& \left\langle B_{p}\right\rangle_{\mathrm{av}} \\
& =\left.\boldsymbol{J}_{7}{ }^{*}\{\boldsymbol{K}(p-1)\}_{1}^{(n-p+2)} \boldsymbol{J}_{p+12}\right|_{0<v_{1}<N_{2}<\cdots<v_{p-1}<n} \sum^{p-1} 1
\end{aligned}
$$

where

$$
\begin{aligned}
& \{\boldsymbol{K}(k)\}_{i}=\left[\begin{array}{cc}
\boldsymbol{K}(k-1) & \boldsymbol{K}(k-1) \boldsymbol{J}_{k+12} \\
\mathbf{0} & 1
\end{array}\right]_{i} \quad(k \geq 3, i \geq 2) \\
& \{\boldsymbol{K}(k)\}_{1}=\boldsymbol{D}_{1} \boldsymbol{I}_{k+5} \quad(k \geq 2) \\
& \boldsymbol{I}_{k v}=\left[\begin{array}{ll}
\boldsymbol{E}_{7} & \mathbf{0}
\end{array}\right]
\end{aligned}
$$

and the order of $\boldsymbol{I}_{k}$ is $7 \times(k+8)$.

Substituting eq 26 and 30 into 22 , we obtain finally

$$
\begin{aligned}
\left\langle S^{2}\right\rangle_{0, \mathrm{a}}= & {\left[p(p-1) \boldsymbol{J}_{7}{ }^{*}\{\boldsymbol{K}(p-1)\}_{1}{ }^{(n-p+2)} \boldsymbol{J}_{p+12}\right.} \\
& \left.-2 p(p-2) \boldsymbol{J}_{p+6}^{*}\{\boldsymbol{H}(p)\}_{1}{ }^{(n-p+1)} \boldsymbol{J}_{p+6}\right](p-1) ! \\
& \times\left[(n+1)^{2} \prod_{i=1}^{p-1}(n-i)\right]^{-1}
\end{aligned}
$$

Substituting 1 or 2 for $p$ in eq 16, we obtain the equation for the linear chain, for which eq 16 corresponds to eq 5 . Similarly, defining $\{\boldsymbol{K}(1)\}_{i}$ by eq 34 ,

$$
\begin{aligned}
& \{\boldsymbol{K}(\mathbf{1})\}_{i}=\left[\begin{array}{ll}
\boldsymbol{D} & \boldsymbol{D} \\
\mathbf{0} & \boldsymbol{D}
\end{array}\right]_{i} \quad(i \geq 2) \\
& \{\boldsymbol{K}(\mathbf{1})\}_{1}=\left[\begin{array}{ll}
\boldsymbol{D}_{1} & \mathbf{0}
\end{array}\right]
\end{aligned}
$$

Substitution of 1 or 2 for $p$ in eq 33 results in the equation for the linear chain, eq 7. Equations 16 and 33 contain no mathematical approximation, and is thus useful to analyse the star-like chains having randomly distributed subchain lengths.

The branching factor $g$ is defined as the ratio of $\left\langle S^{2}\right\rangle_{0, \text { av }}$ of star-like chain to $\left\langle S^{2}\right\rangle_{0}$ of the linear chain whose number of bond is identical with that of the branched chain. In the case of star-like chains having uniformly distributed subchain length, the branching factor $g$ is obtained by dividing eq 11 by eq 6 or dividing eq 12 by eq 7 . Then, the following equation is obtained as the $g$ value for infinite degree of polymerization.

$$
g=\frac{3 p-2}{p^{2}}
$$

This equation is in accord with the results obtained by Zimm and Stockmayer, ${ }^{1}$ and Kurata and Fukatsu' ${ }^{2}$ for the Gaussian chain. Similarly, in the case of star-like chain having randomly distributed subchain lengths, the branching factor $g$ is obtained by diving eq 16 by eq 6 or dividing eq 33 by eq 7 . Then, the following equation is obtained for the infinite degree of polymerization,

$$
g=\begin{gathered}
6 p \\
(p+1)(p+2)
\end{gathered}
$$

This equation also agrees with Zimm's and Kurata's result.

\section{NUMERICAL RESULTS AND DISCUSSION}

The characteristic ratio $\left\langle R^{2}\right\rangle_{0} / n l^{2}$ and the radius of the gyration ratio $\left\langle S^{2}\right\rangle_{0} / n l^{2}$ of linear polypeptides were calculated by Flory, et al., ${ }^{4,5}$ and Tanaka and Nakajima. ${ }^{10,11}$ It was mentioned in these papers that the value of the van der Waals radius $r_{\mathrm{CH}_{2}}$ for $\beta$-methylene group significantly affected the calculated value of the characteristic ratio and the radius of gyration ratio, and that linear side chains longer than methyl should have little influence on the characteristic ratio and the radius of gyration ratio. Thus the results calculated for poly-L-alanine may be applied to other polypeptides composed of $\alpha$-Lamino acid residues. Consequently, the calculations in this study are carried out for star-like polypeptides composed of L-alanine residues. 
Flory and his co-workers ${ }^{5}$ arrived at the following matrix for poly-L-alanine.

$$
\langle T\rangle=\left[\begin{array}{crr}
0.51 & 0.20 & 0.59 \\
-0.046 & -0.61 & 0.21 \\
0.65 & -0.23 & -0.30
\end{array}\right]
$$

This formula is used in our calculation.

\section{The Radius of Gyration Ratio}

Figure 2 (uniform distribution) and Figure 3 (random distribution) indicate that the radius of the gyration ratio $\left\langle S^{2}\right\rangle_{0, \mathrm{av}} / n l^{2}$ of star-like molecules whose branch lengths distribute uniformly and randomly, increase asymptotically with the degree of polymerization $n$ like that of linear chain. This tendency is remarkable in the case of random distribution of subchain length. Figures 2 and 3 also show that $\left\langle S^{2}\right\rangle_{0, \text { av }} / n l^{2}$ of star-like chains always smaller than that of linear chain of identical $n$. Figure 4 indicates that $\left\langle S^{2}\right\rangle_{0, \text { av }} / n l^{2}$ decreases asymptotically with number of branches $p$, and $\left\langle S^{2}\right\rangle_{0, \text { av }} / n l^{2}$ of random distribution is always larger than that of uniform

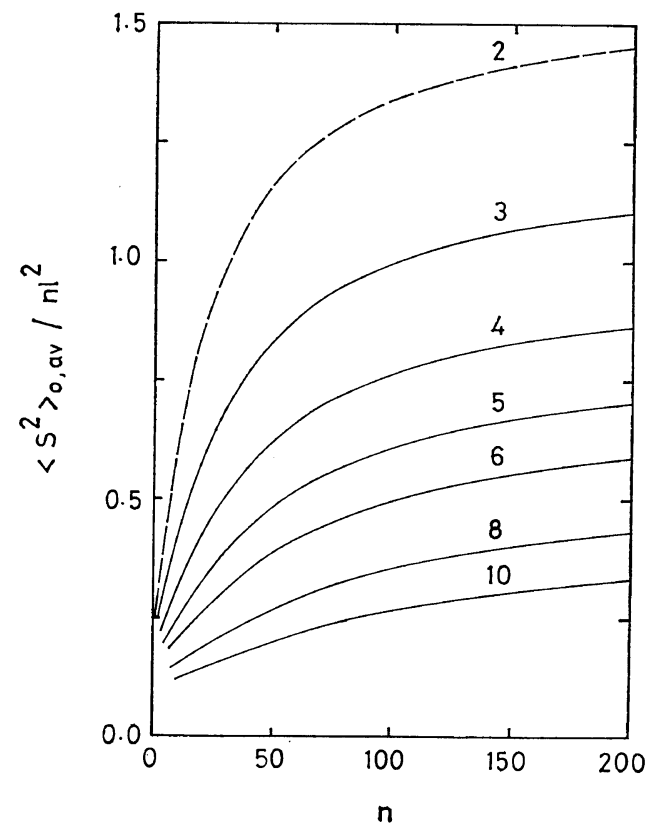

Figure 2. The radius of the gyration ratio $\left\langle S^{2}\right\rangle_{0, \text { av }} / n l^{2}$ plotted against the degree of polymerization $\boldsymbol{n}$ for star-like polypeptides having uniform distribution of subchain length. The numeral on each curve indicates the number of branches.

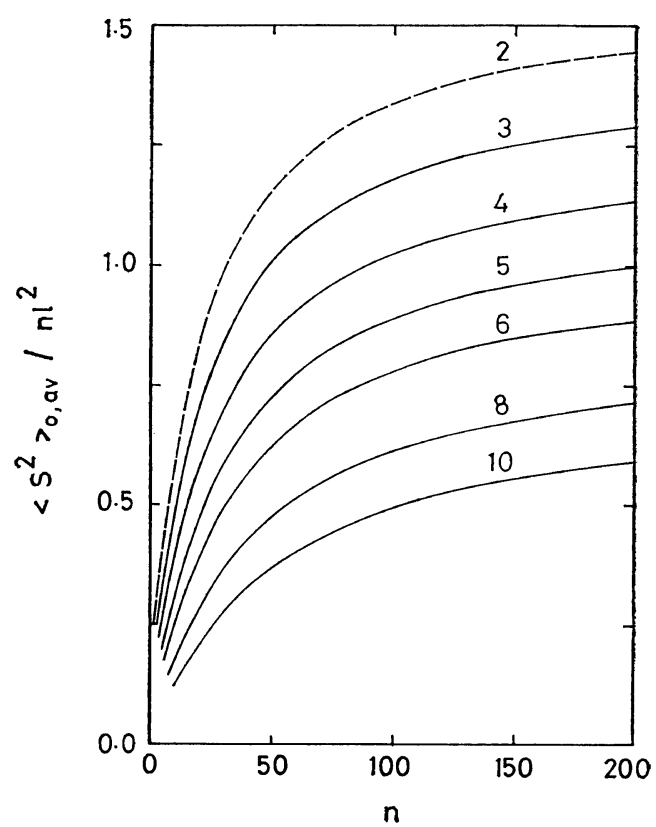

Figure 3. The radius of the gyration ratio $\left\langle S^{2}\right\rangle_{0, \text { av }} / n l^{2}$ plotted against the degree of polymerization $n$ for star-like polypeptides having random distribution of subchain length. The numeral on each curve indicates the number of branches.

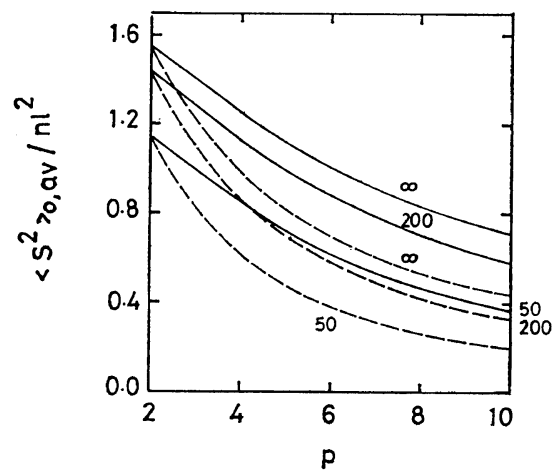

Figure 4. The radius of the gyration ratio $\left\langle S^{2}\right\rangle_{0, \text { av }} / n l^{2}$ plotted against the number of branches $p$ for star-like polypeptides having random distribution of subchain length (solid lines), and having uniform distribution of subchain length (broken lines). The numeral on each curve indicates the degree of polymerization.

distribution of identical $p$. Also, Figure 5 indicates that $r_{s}$, which is the ratio $\left\langle S^{2}\right\rangle_{0, \text { av }} / n l^{2}$ to $\left(\left\langle S^{2}\right\rangle_{0, \mathrm{av}} / n l^{2}\right)_{\infty}\left(\left(\left\langle S^{2}\right\rangle_{0, \mathrm{av}} / n l^{2}\right)_{\infty}\right.$ is the asymptotic value of $\left\langle S^{2}\right\rangle_{0, \text { av }} / n l^{2}$ at $\left.n\right)$, decreases asymp- 
Unperturbed Chain Dimension of Branched Polypeptides. I.

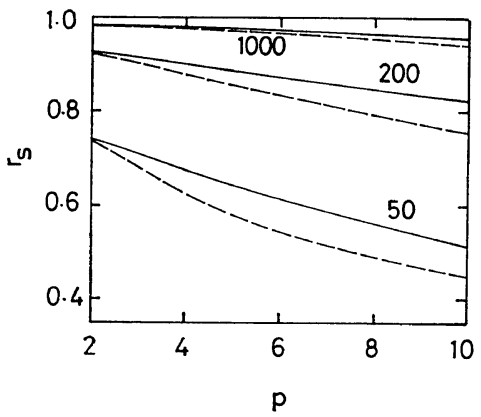

Figure 5. $\boldsymbol{r}_{s}$, which is the ratio $\left\langle\boldsymbol{S}^{2}\right\rangle_{0, \mathrm{av}} / n l^{2}$ to $\left(\left\langle S^{2}\right\rangle_{0, a v} / n l^{2}\right)_{\infty}$, plotted against the number of branches $p$ for star-like polypeptides having random distribution of subchain length (solid lines), and having uniform distribution of subchain length (broken lines). The numeral on each curve indicates the degree of polymerization.

Table I. Summary of the number of bonds required for $\left(\left\langle S^{2}\right\rangle_{0, \mathrm{av}} / n l^{2}\right)_{\infty}-\left\langle S^{2}\right\rangle_{0, \mathrm{av}} / n l^{2}$ to be within 10 and $5 \%$ of $\left(\left\langle S^{2}\right\rangle_{0, \mathrm{av}} / n l^{2}\right)_{\infty}$ for star-like polypeptides

\begin{tabular}{rcccc}
\hline & \multicolumn{2}{c}{ Uniform } & \multicolumn{2}{c}{ Random } \\
\hline 2 & $n(10 \%)$ & $n(5 \%)$ & $n(10 \%)$ & $n(5 \%)$ \\
\hline 3 & 157 & 330 & 157 & 330 \\
4 & 201 & 414 & 177 & 370 \\
5 & 248 & 508 & 205 & 420 \\
6 & 342 & 576 & 255 & 530 \\
8 & 440 & 896 & 315 & 640 \\
10 & 540 & 1100 & 370 & 760 \\
\hline
\end{tabular}

totically with the number of branches $p$, and $r_{s}$ of random distribution is always larger than that of uniform distribution of identical $p$. Table $I$ indicates that the number of bonds required for $\left(\left\langle S^{2}\right\rangle_{0, \text { av }} / n l^{2}\right)_{\infty}-\left\langle S^{2}\right\rangle_{0, \text { av }} / n l^{2}$ to approach to within 10 and $5 \%$ of $\left(\left\langle S^{2}\right\rangle_{0, \text { av }} / n l^{2}\right)_{\infty}$ is always larger for uniform distribution than for random distribution, and that the number of these increases with an increasing number of branches $p$. Figure 6 represents each term of eq 16 as functions of the degree of polymerization for $p=3$. The 1 st term in braces of eq 16 is represented by $A_{1}$, and the 2 nd and 3 rd terms are by $A_{2}$. The 4 and 5 th terms are, respectively, represented by $B_{1}$ and $B_{2}$. The term for $i=k$ in the 6th term are represented by $C_{k+4}$. The terms $B_{1}, B_{2}, C_{5}$, and $C_{6}$ have a large influence



Figure 6. Each term of eq 16 calculated for $p=3$ as functions of the degree of polymerization. The 1 st term within the braces of eq 16 is represented by $A_{1}$, and the 2 nd and 3 rd terms by $A_{2}$. The 4th and 5 th, terms are, respectively, represented by $B_{1}$ and $B_{2}$. The terms for $i=k$ in the 6 th term are represented by $C_{k+4}$. The broken line indicates $\left\langle S^{2}\right\rangle_{0, \text { av }} / n l^{2}$.

on $\left\langle S^{2}\right\rangle_{0, \text { av }} / n l^{2}$ at $n<20$, but converge very rapidly to 0 with increasing $n$. The term $A_{2}$ converges slowly. The effect of this term cannot be neglected for a large value of $n$.

Branching Factor

Zimm and Stockmayer, ${ }^{1}$ and Kurata and Fukatsu $^{2}$ showed that the branching factor $g$ of star-like chains whose branch lengths distribute uniformly and randomly is dependent on the numbers of branches $p$. In this work Figures 7-9 show that the branching factor of star-like chain dependents not only on the number of branches $p$, but also the degree of polymerization $n$. In the case of random distribution, the value of $g$ increases monotonously to the asymptotic limit, i.e., $g_{\infty}$, while, in the case of uniform distribution, the value of $g$ decreases with $n$, and passes through a minimum, i.e., $g_{\mathrm{min}}$, and increases with $n$ to the value of $g_{\infty}$. In the case of uniform distribution for $p=3$, the value of $g$ reaches a minimum value $g_{\min }=$ 0.685 when $n=15$; the value of $g_{\min }$ decreases with $p$, that is, $g_{\min }=0.499$ when $n=16$ for $p=4$, 
and $g_{\min }=0.163$ when $n=30$ for $p=10$. The value of $g$ converges more rapidly to $g_{\infty}$ with increasing $n$ than $\left\langle S^{2}\right\rangle_{0, \text { av }} / n l^{2}$ in both cases of uniform and random distributions. Table II shows that the number of bonds required for $g_{\infty}-g$ to approach to within 10 and $5 \%$ of $g_{\infty}$ is always larger for uniform distribution than for random distribution, and that the number of these increases with an increasing number of branches $p$.

From the results mentioned above, the radius of the gyration ratio $\left\langle S^{2}\right\rangle_{0, \text { av }} / n l^{2}$ and the branching factor $g$ depend not only on the number of branches $p$ but also on the degree of polymerization $n$. These two parameters are important factors for the characterization of star-like polypeptides as a model of globular proteins.

Tonelli $^{12}$ has calculated the value of $g$ for the particular star-like and comb-like polyethylenes whose subchain length is specified. In the case of a 3 branched star-like chain whose subchain lengths $n_{k}$ are 25, 50, and 100, Tonelli. obtained the values of $0.737,0.769$, and 0.776 for $g$,

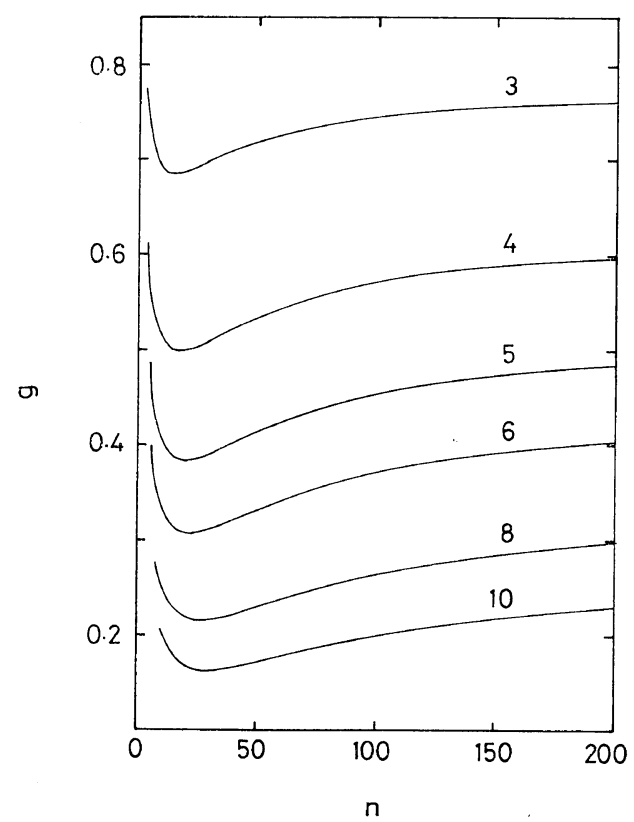

Figure 7. The branching factor $g$ plotted against the degree of polymerization $n$ for star-like polypeptides having uniform distribution of the subchain length. The numeral on each curve indicates the number of branches.

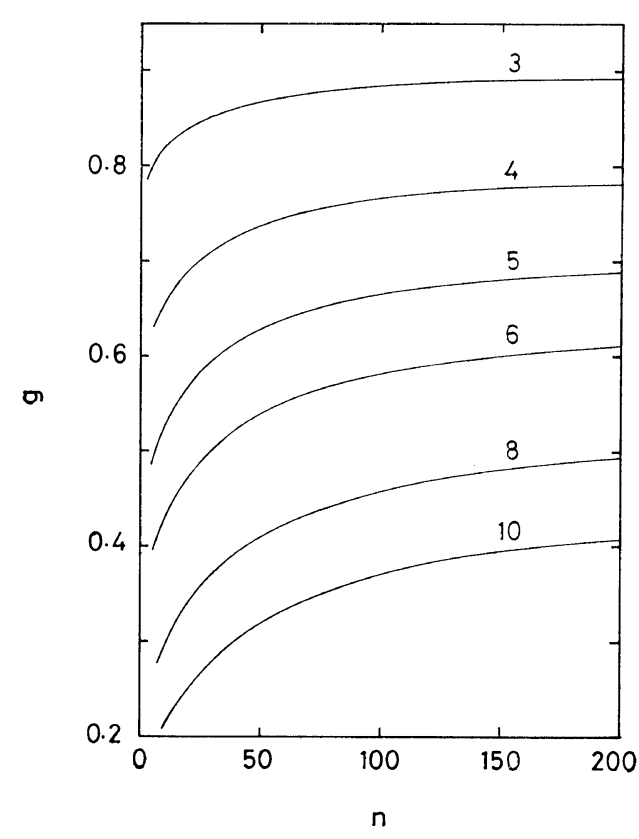

Figure 8. The branching factor $g$ plotted against the degree of polymerization $n$ for star-like polypeptides having random distribution of the subchain length. The numeral on each curve indicates the number of branches.

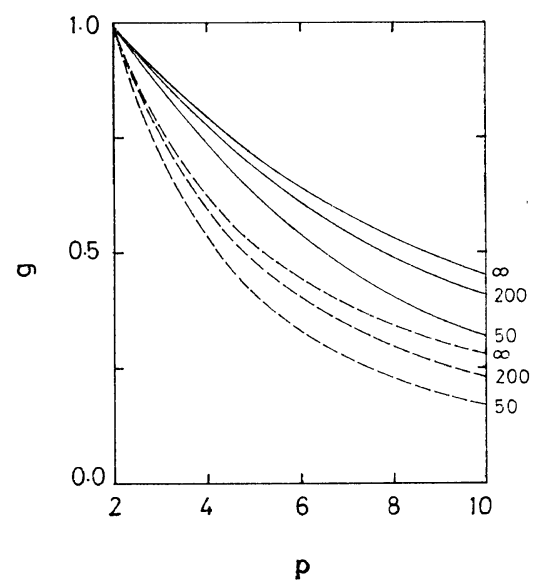

Figure 9. The branching factor $g$ plotted against the number of branches $p$ for star-like polypeptides having random distribution of subchain length (solid lines), and having uniform distribution of the subchain length (broken lines). The numeral on each curve indicates the degree of polymerization. 
Unperturbed Chain Dimension of Branched Polypeptides. I.

respectively. These results are slightly larger than our results $0.735,0.756$, and 0.767 . In the case of a 4 branched star-like chain, his results are $g=0.568,0.615$ and 0.608 , for $n_{l k}=25,50$, and 100 . These results are slightly smaller than our results 0.571 and 0.611 for $n_{k}=25$ and 100, and slightly larger than our result 0.597 for $n_{k}=50$. Though his configuration partition function was formulated incorrectly and the polymers used as the model were different, the difference between the two value of $g$ is smaller than that between $g_{\infty}$ and $g$ for the corresponding $n$.

Recently, Mattice and his coworker ${ }^{13-15}$ have calculated values of $g$ for the 3 and 4 branched star-like polyethylenes whose subchain lengths are uniformly distributed. Their value for $g_{\text {min }}$

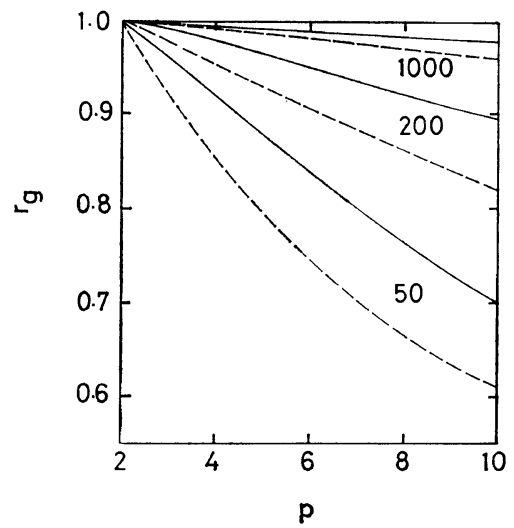

Figure 10. The ratio $g$ to $g_{\infty}$ plotted against the number of branches $p$ for star-like polypeptides having random distribution of subchain length (solid lines), and having uniform distribution of the subchain length (broken lines). The numeral on each curve indicates the degree of polymerization.

Table II. Summary of the number of bonds required for $g_{\infty}-g$ to be within 10 and $5 \%$ of $g_{\infty}$ for star-like polypeptides

\begin{tabular}{rrrrr}
\hline & \multicolumn{2}{c}{ Uniform } & \multicolumn{2}{c}{ Random } \\
\cline { 2 - 5 } & $n(10 \%)$ & $n(5 \%)$ & $n(10 \%)$ & $n(5 \%)$ \\
\hline 3 & 33 & 84 & 8 & 34 \\
4 & 84 & 180 & 37 & 87 \\
5 & 135 & 280 & 65 & 142 \\
6 & 186 & 378 & 93 & 249 \\
8 & 280 & 576 & 150 & 315 \\
10 & 380 & 770 & 208 & 430 \\
\hline
\end{tabular}

Table III. Summary of the results on $g$ for starlike chains whose subchain lengths distribute uniformly

\begin{tabular}{rlll}
\hline$p$ & $n\left(g_{\min }\right)^{\mathrm{a}}$ & \multicolumn{1}{c}{$g_{\min }$} & $n(1 \%)^{\mathrm{b}}$ \\
\hline 3 & $15(15)^{\mathrm{c}}$ & $0.685(0.659)$ & $432(650)$ \\
4 & $16(16)$ & $0.499(0.480)$ & $904(1200)$ \\
5 & 20 & 0.383 & 1390 \\
6 & 24 & 0.308 & 1878 \\
8 & 24 & 0.216 & $2500<$ \\
10 & 30 & 0.163 & $2500<$ \\
\hline
\end{tabular}

a Number of bonds at which $g$ attains the minimum value $g_{\mathrm{min}}$.

b Number of bonds required for $g_{\infty}-g$ to be within $1 \%$ of $g_{\infty}$.

c Numerical values in parentheses are Mattice's results obtained with $\sigma=0.54, \phi=1$, and $\omega=0.088$ for the 3 and 4 branched star-like polyethylenes.

was slightly smaller than our results, but the number of bonds for $g_{\min }$ was identical with our results. The number of bonds required for $g_{\infty}-g$ within $1 \%$ of $g_{\infty}$, which they have obtained, are larger than our results. That is, the convergence of the $g$ value to the limiting value $g_{\infty}$ is slower than that for star-like polyethylenes, but the difference between their results and ours is very small.

Computations were carried out by the FACOM 230-75 computer at the Kyoto University Computation Center.

\section{APPENDIX}

\section{Modification of the Lagrange's Theorem}

For the star-like chain as shown in Figure 1, if each atom is equal in mass, the square radius of gyration $S^{2}$ is represented by eq 1 . Let the vector from the branching point 0 to the $i$-th atom be $r_{0 i}$, and then

$$
S_{i}=S_{0}+\boldsymbol{r}_{0 i}
$$

From eq 1 and A1 we obtain the following relation

$$
S^{2}=S_{0}{ }^{2}+\frac{2}{n+1} S_{0} \sum_{k=1}^{p} \sum_{i=1}^{n_{k}} \boldsymbol{r}_{0 i}+\frac{1}{n+1} \sum_{k=1}^{p} \sum_{i=1}^{n_{k}} r_{0 i}^{2}
$$

The definition of $S_{i}$ leads to

$$
\sum_{k=1}^{p} \sum_{i=1}^{n_{k}} \boldsymbol{S}_{i}+\boldsymbol{S}_{0}=\mathbf{0}
$$


By substituting eq $A 1$ into $A 3$, we obtain the following equation.

$$
\boldsymbol{S}_{0}=-\frac{1}{n+1} \sum_{k=1}^{p} \sum_{i=1}^{n_{k}} \boldsymbol{r}_{0 i}
$$

Combination of eq A2 with A4 results in

$$
\begin{aligned}
S^{2}= & \frac{1}{n+1} \sum_{k=1}^{p} \sum_{i=1}^{n_{k}} r_{0 i}^{2}-\frac{1}{(n+1)^{2}} \sum_{k=1}^{p} \sum_{i=1}^{n_{k}} \sum_{j=1}^{n_{h}} \boldsymbol{r}_{0 i} \boldsymbol{r}_{0 j} \\
& -\frac{2}{(n+1)^{2}} \sum_{1 \leqq k<h \leqq p} \sum_{i=1} \sum_{j=1}^{n_{k}} \boldsymbol{r}_{0 i} \boldsymbol{r}_{0 j}
\end{aligned}
$$

Finally substituting the cosine rule

$$
\boldsymbol{r}_{0 i} \boldsymbol{r}_{0 j}=\frac{1}{2}\left(r_{0 i}^{2}+r_{0 j}^{2}+r_{i j}^{2}\right)
$$

into eq $\mathrm{A} 5$, eq 2 is obtained.

\section{REFERENCES}

1. B. H. Zimm and W. H. Stockmayer, J. Chem. Phys., 17, 1301 (1949).

2. M. Kurata and M. Fukatsu, ibid., 41, 2934 (1964).
3. P. J. Flory, "Statistical Mechanics of Chain Molecules," John Wiley \& Sons, Inc., New York, N.Y., 1969.

4. D. A. Brant and P. J. Flory, J. Am. Chem. Soc., 87, 2791 (1965).

5. D. A. Brant, W. G. Miller, and P. J. Flory, J. Mol. Biol., 23, 47 (1967).

6. P. J. Flory, Macromolecules, 7, 381 (1974).

7. P. J. Flory and R. L. Jernigan, J. Chem. Phys., 42, 3509 (1965).

8. P. J. Flory, Proc. Natl. Acad. Sci. U.S., 70, 1819 (1973).

9. P. J. Flory and Y. Abe, J. Chem. Phys., 54, 1351 (1971).

10. S. Tanaka and A. Nakajima, Polym. J., 1, 71 (1970).

11. S. Tanaka and A. Nakajima, ibid., 2, 717 (1971).

12. A. E. Tonelli, J. Am. Chem. Soc., 94, 2972 (1972).

13. W. L. Mattice, Macromolecules, 8, 644 (1975).

14. W. L. Mattice, ibid., 9, 48 (1976).

15. W. L. Mattice and D. K. Carpenter, ibid., 9, 53 (1976). 